\title{
MOTION OF THE CORE, AND ITS \\ INFLUENCE ON THE EARTH'S AXIS
}

\author{
JOSE MATEO
}

La Plata Astronomical Observatory, La Plata, Argentina

\begin{abstract}
After the advent of artificial Earth satellites, an accurate knowledge of the harmonic coefficients of the Earth's potential has enabled us to determine the size and shape of the Earth with extraordinary accuracy.

The gravitational force between the core and the rest of the Earth, which makes both centers of mass tend to coincide, is so enormous that there is a strong possibility of a very slow motion of the core forcing its way into the mantle. It includes secular variations in both latitude and the time of rotation of the Earth.
\end{abstract}

\section{Introduction}

The areal variations of the geoid have brought out some relevant facts for geophysical studies, namely:

(1) At the extended plateau of Pamir in central Asia, it would be expected that the geoid would run outside the reference spheroid because of the high mean elevation of $4000 \mathrm{~m}$. Instead, it was found to run $60 \mathrm{~m}$ below the mean spheroid. It is also known that if we condense the plateau to the spheroid level, the geoid would shift only $80 \mathrm{~cm}$ (Pizzetti, 1913).

Similarly, the wide-spread continent of Antarctic, with a mean elevation of $2000 \mathrm{~m}$, we should expect the geoid to be located outside the reference spheroid; but there, too, it was found to be approximately $30 \mathrm{~m}$ below.

(2) In contrast with the Pamir and Antarctic as for areas such as the Arctic, Atlantic and Pacific Oceans, where external masses are negligible the geoid was found to be outside the spheroid by as much as $60 \mathrm{~m}$ rather than below, as one would expect.

The external masses alone do not explain these anomalous conditions so it is believed that internal factors are present, strongly suggesting that these conditions are caused by the core's center of mass not coinciding with that of the Earth.

\section{Position of the Center of Mass of the Core}

As shown in Figure 1, the equatorial plane has an elevation of $60 \mathrm{~m}$ at $130^{\circ}$ East longitude (western Pacific) and a depression of $30 \mathrm{~m}$ at $50^{\circ}$ West longitude (Atlantic and NE Brazil). This effect is mainly produced by the harmonic coefficient $J_{33}$ which creates the 'equatorial pear shape'. Considering the meridian at $50^{\circ}$ West longitude as the origin of the angles, the potential difference between the two positions can be determined. If the center of the core is displaced, it should also produce a difference of potentials between the two positions. Assuming that the geoidal potential difference is produced by the out of center position of the core, it follows that the displacement 


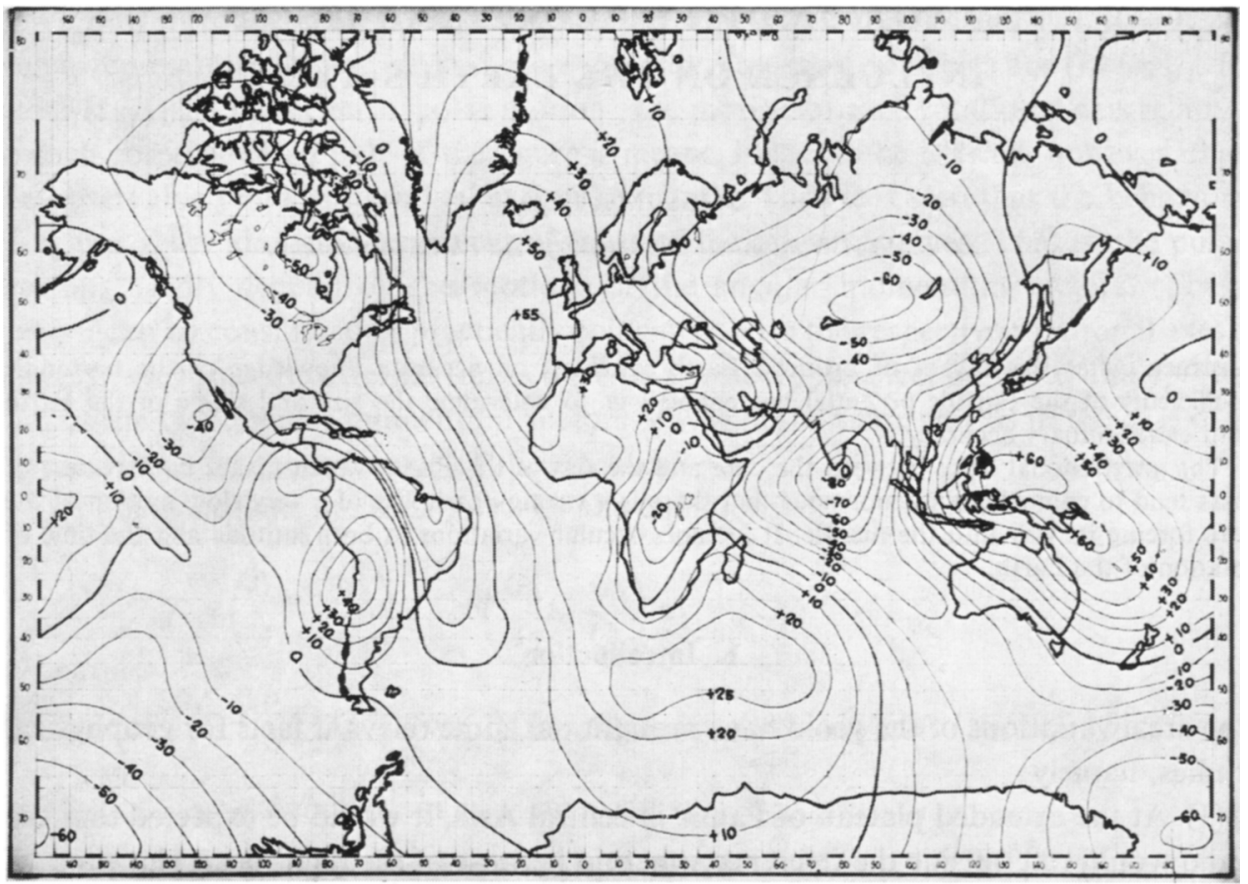

Fig. 1.

is approximately $290 \mathrm{~m}$. This value is verified by using the harmonic coefficient $J_{22}$ which is mainly responsible for the equatorial ellipticity. Assuming once again that the geoidal potential difference is produced by the out of center position of the core, the result is again a displacement of $290 \mathrm{~m}$.

The 'pear shape' of the Earth is mainly produced by the harmonic coefficient $J_{3}$ which results in different flattening of the hemispheres. Using this coefficient, the difference between the geoidal potentials at the North and South Poles can be determined. As before, if the core is displaced by an unknown amount in a vertical plane, that potential difference may also be determined, and assuming once again that these differences are equal, the displaced distance was calculated as approximately $90 \mathrm{~m}$ northward. If the center of mass of the Earth as a whole is used as an origin, the distance between the centers of mass of the core and the mantle-crust was found to be $460 \mathrm{~m}$ (Birch, 1964; Kaula, 1967; Kosai, 1969).

\section{Gravitational Force Between the Core and the Mantle-crust}

The gravitational force which makes the centers of mass of the core and mantle-crust tend to coincide has been computed as $11.4 \times 10^{25} \mathrm{cgs}$. It should be emphasized that this force is six times greater than that needed to keep the Moon in orbit.

Because of the compressional stress on the lower mantle and the stress displacing the mantle material in opposite directions, continental masses like Europe and Ameri- 


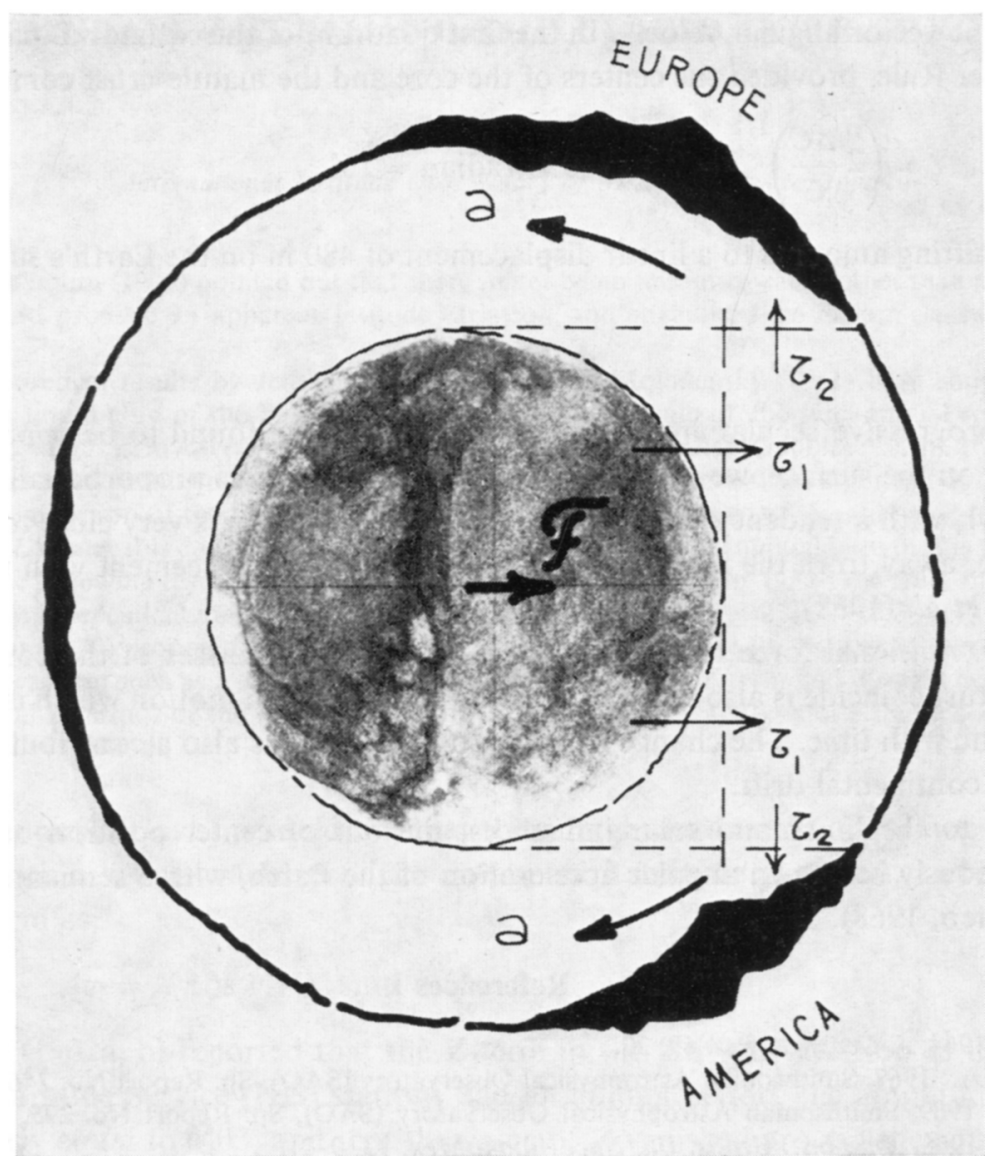

Fig. 2.

ca could be moved, as shown in Figure 2. This well-known continental drift can also be related to the motion of the core.

If a secular motion of the core exists, the position of the vector angular momentum must also change secularly owing to a very slow shift of the center of mass of the core. Since astronomers have proved that the separation between this vector and the vector angular velocity is negligible, we can assume them to coincide (Vicente, 1964). By also assuming that the vector coincides with the $Z$-axis of a reference coordinate system $(X, Y, Z)$, the direction cosines were found to be

$$
\xi=\frac{\Delta E}{A-C} ; \quad \eta=\frac{\Delta F}{B-C} ; \quad \zeta=1-\frac{\Delta C}{C} .
$$

As it has been established that the inertia axis of the Earth and the rotation axis do not coincide, then the values of the products of inertia $\Delta E$ and $\Delta F$ must be negative. Since $(A-C)$ and $(B-C)$ are also negative, the direction cosines $\xi$ and $\eta$ are positive, 
placing the vector angular velocity in the first quadrant of the equatorial plane. Using the Steiner Rule, provided the centers of the core and the mantle-crust coincide

$$
\zeta=\left(2 \frac{\Delta C}{C}\right)^{1 / 2}=7.8 \times 10^{-5} \text { radian }=16^{\prime \prime} .
$$

This shifting amounts to a linear displacement of $480 \mathrm{~m}$ on the Earth's surface.

\section{Conclusions}

As the progressive secular motion of the pole has been found to be approximately $10 \mathrm{~cm} / \mathrm{yr}$ on the surface, we may also say that the core moves proportionally at a rate of $6 \mathrm{~cm} / \mathrm{yr}$, with a tendency to diminish. This secular motion is very close to $50^{\circ}$ West longitude, away from the mean pole. This result is also in agreement with the results of Yumi et al. (1968).

The gravitational force tending to make the center of masses of the core and the mantle-crust coincide is also decreasing, and this results in a motion which is evidently asymptotic with time. The change of position of the core is also a contributory factor affecting continental drift.

Owing to the lunar and solar influences, the out of center position of the core simultaneously brings an angular acceleration of the Earth, with a semiannual variation (Mateo, 1968).

\section{References}

Birch, F.: 1964, J. Geophys. Res. 69, 20.

Kaula, W. A.: 1967, Smithsonian Astrophysical Observatory (SAO), Sp. Report No. 246, July 1967. Kosai, Y.: 1969, Smithsonian Astrophysical Observatory (SAO), Sp. Report No. 295, Febr. 1969. Mateo, J.: 1968, Ann. Inst. Geofis. Un. Nac. Auton. Mex. 13, 9-21.

Pizzetti, P.: 1913, in E. Spoerri (ed.), Principii della Teoria Mecanica della Figura dei Pianeti, Pisa, p. 28.

Vicente, R. O.: 1964, 'The Influence of the Core on Earth Tides', Comm. No. 236, Cinquime Symp. Intern. sur les Marées Terrestres, Bruxelles, p. 43.

Yumi, S., Wakō, Y., and Markowitz, Wm.: 1968, in Wm. Markowitz and B. Guinot (eds.), 'Continental Drift, Secular Motion of the Pole, and Rotation of the Earth', IAU Symp. 32, 33.

\section{DISCUSSION}

J. A. Jacobs: Why do you make the problem so complicated - why not account for differences in height of the geoid by lateral inhomogeneities in the mantle - even as deep as the mantlecore boundary if you wish (Compare suggestion of Hide)?

J. Mateo: I have made an effort to simplify the problem to the best of my ability - As to using inhomogeneities in the mantle for comparison, it could only cover a small fraction of my research owing to the tremendous mass of the core. 